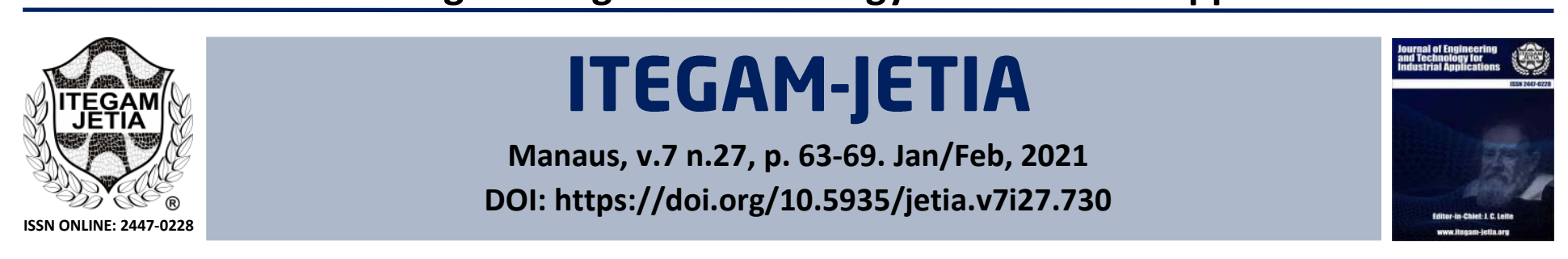

\title{
OPEN-SOURCE SOFTWARE IN EMERGING TECHNOLOGIES FOR ECONOMIC GROWTH
}

\author{
Eseosa Ehioghae*1 and Sunday Idowu ${ }^{2}$ \\ ${ }^{1,2}$ Department of Software Engineering, School of Computing and Engineering Sciences, Babcock University. Ilishan-Remo, Ogun State, Nigeria.
}

${ }^{1}$ http://orcid.org/0000-0003-4890-5303 (1), ${ }^{2}$ http://orcid.org/0000-0002-0013-0265 (1)

Email: *ehioghaees@babcock.edu.ng, idowus@babcock.edu.ng

\section{ARTICLE INFO}

\section{Article History}

Received: December $10^{\text {th }}, 2020$

Accepted: January $28^{\text {th }}, 2021$

Published: February $15^{\text {th }}, 2021$

\section{Keywords:}

Open-Source,

Emerging Technology,

Economic Growth,

Disruption,

Creative Destruction.

\begin{abstract}
The growth of the economy is one of the aims of every country, as it ensures an improved standard of living for the citizens of such countries. One of the ways this economic growth can be achieved is by investing in technologies that can yield more efficiency in operations while reducing overall costs. The developed nations have more economic prowess because of their heavy investment in technological development, which sometimes could be elusive in developing nations. Open-source software, however, levels the playing ground as it presents strengths that can drive innovation faster than can be achieved with the proprietary software development paradigm. Emerging technologies on the other hand present transformative powers that can catalyze the economic growth of the countries that can efficiently apply them. This paper examines the key characteristics of open-source software alongside emerging technologies to establish the impact of open-source software on emerging technologies and its wider impact on economic growth. Specific emerging technologies where open-source concepts are being applied were examined to reveal the positive impact of open-source software, while at the same time, specific emerging technologies that are creating disruptions that drive economic growth were investigated. It was finally concluded that open-source software presents key strengths, which when combined with the disruptive powers of emerging technologies, presents tremendous potential to increase the economic growth of the country in which it is implemented.
\end{abstract}

\section{INTRODUCTION}

Open-Source Software (OSS) is premised on the idea of open innovation, where collaboration, knowledge exchange, and volunteer software development foster innovation in the production of software applications. OSS specifically presents the idea that open software is more effective than propriety software given that OSS provides excellent opportunities for pertinent collaboration and knowledge sharing, which results in a net positive effect of allowing software to be produced by leveraging the knowledge and expertise of a wider open community more effectively, in contrast to the proprietary software development approaches which takes a more closed approach.

Emerging technologies have been a crucial area of research interest, both in academia and industry. Albeit not having a specific definition as to which technology is emergent, various researchers have come up with definitions that attempt to describe what emerging technologies are [1-3]. Rotolo, Hicks, and Martin [1] were able to identify five key characteristics that classify a technology as emergent. They include (i) radical novelty, (ii) relatively fast growth, (iii) coherence, (iv) prominent impact, and (v) uncertainty and ambiguity. Furthermore, they gave a more specific definition of emerging technology as "a radically novel and relatively fast growing technology characterized by a certain degree of coherence persisting over time and with the potential to exert a considerable impact on the socio-economic domain(s) which is observed in terms of the composition of actors, institutions and patterns of interactions among those, along with the associated knowledge production processes. Its most prominent impact, however, lies in the future and so in the emergence phase is still 
somewhat uncertain and ambiguous" [1]. One can readily identify disruptive technologies such as Artificial Intelligence, the Internet of Things (IoT), Blockchain, Autonomous Devices as examples of technologies that can fall within the categories of technologies called emerging technology.

The intrinsic nature of knowledge sharing and collaboration present in OSS can greatly contribute to emerging technologies. Novel ideas could be birthed from the openness of OSS, which could also yield a relatively fast growth of the technology, which in contrast could never be achieved within the closed walls of proprietary software paradigms.

The economic growth of every nation on the other hand is a key factor in measuring the success of such a nation. The United States, for example, is the world's leading nation because of the high level of economic growth present in the country. Knowledge and collaboration play a huge role in the growth of any economy. Ghafele and Gibert [4] identified knowledge and the correct utilization of technology as a crucial indicator of national growth. Hence, the right application of technology with knowledge sharing and collaboration presents an appropriate clime for innovation, which fortunately are the tenets on which OSS rests on.

This research, therefore, identifies the positive impacts of OSS within emerging technologies, which can catalyze the economic growth of any nation in which OSS paradigms are adopted.

\section{OPEN-SOURCE SOFTWARE LANDSCAPE}

As has now been established, OSS presents certain characteristics which can act as a catalyst for innovation. However, crucial to understanding the impacts of OSS in the economic development of any nation is the understanding of what exactly OSS entails.

\section{II.1 WHAT IS OPEN-SOURCE SOFTWARE}

OSS is another name for a broader classification of software which is called Free and Open-Source Software (FOSS). These are software that can be freely used, modified, and distributed with respect to the copyright and license of its open-source status [5, 6]. This implies that Open-Source Software generally have some licenses that dictate how the code should be freely used and distributed. For example, some open-source license mandates that the user altering and redistributing the software must also share the source code of the software and at no cost [7].

OSS today is a major pillar for innovation and ideas that birth new products faster than the in the closed walls of proprietary software development. Ebert [8] identified software technologies and processes as the most prominent areas in which open source innovations are taking place.

Software technologies have seen massive improvements and innovations since the adoption of OSS. The positive impacts of software technologies such as Apache, Linux, MySQL cannot be overemphasized as the momentum in innovation provided by these technologies is self-evident in our everyday interaction with software products. This momentum in software technologies is as a result of OSS leveraging a vast pool of developer expertise, alongside collaboration with the community, to ensure that software products produced are highly efficient, more secure, and with lesser bugs. Eric Raymond captured this more accurately when he said "given enough eyeballs, all bugs are shallow" [9].

In addition, software processes, which lead to the production and maintenance of software products, have been greatly improved due to the existence of OSS. Agile development techniques, distributed change management, and global collaboration are just a few of the processes that have revolutionized software development. This, as Ebert [8] intuited, has been because software developers incorporated these processes in their open-source projects, which, over time, saw positive impacts in the development process, after which gained massive adoption within the global software community.

\section{EMERGING TECHNOLOGIES LANDSCAPE}

As previously established, emerging technologies are a little difficult to readily define [1-3]. Avila-Robinson and Miyazaki [10] further clarified this by referring to the fact that attempting to give a single definition of emerging technologies has led to numerous terminologies, with overlapping concepts, which still points to the intrinsic difficulty in defining emerging technologies. Halaweh [2] also alluded to this difficulty by saying that some technologies might be considered emerging in some contexts while in some other contexts considered as established. A good example given by Halaweh [2] was Radio Frequency Identification (RFID), which is not considered as emerging in developed countries, given its widespread use, but considered emerging in many developing countries, given its low diffusion in such countries as a result of the lack of the underlying infrastructure to support it. Furthermore, Day and Schoemaker [3] defined emerging technologies as "science-based innovations that have the potential to create a new industry or transform an existing one" [3]. Adner and Levinthal [11] further hinted at this when they divided emerging technologies pattern into two broad categories of lineage development which involves a technology adapting to the particular needs of the niche it is being applied to, and creative destruction, which involves a new technology emerging from an existing one by destroying the techniques of the existing one, paving way for more efficient techniques.

As already established, the nature of emerging technologies is quite hard to qualify, as technologies considered as emerging technologies in developing countries might have plateaued in developed countries, due to their widespread implementation in the developed countries. Atkinson [12] identified a new wave of technological emergence that, although currently implemented in some industry verticals, still lack a global adoption, due to their nascent nature and the current costs involved in implementing them on a large scale. Some of these technologies include Artificial Intelligence, The Internet of Things, Blockchain, Autonomous Devices, Robotics. These are good examples of the technologies that fall under the emerging technologies umbrella based on the categorization given by Rotolo, Hicks, and Martin [1].

It should be noted that a high rate of innovation can be achieved by the strengths of OSS and emerging technologies [13] which can lead to an increase in the standard of living, and the overall economic development of countries by providing numerous job opportunities [12]. We now explore how OSS and emerging technologies lead to economic growth.

\section{OPEN SOURCE SOFTWARE AND EMERGING TECHNOLOGIES AS A FORCE FOR DISRUPTION AND ECONOMIC GROWTH}

OSS and emerging technologies are a great force for disruption and economic growth. Various studies have been conducted to show the net positive effects of OSS and emerging technologies in economic growth. In a study by Ghafele and Gibert [4] examining the impact of Open-Source Software on employment in the USA, it was discovered that the open innovation intrinsic to 
OSS yielded employment growth with a positive impact in highincome jobs across various economic sectors in the United States, for a projection period of 10 years from 2008 to 2018. Garzarelli, Limam, and Thomassen [14] also intuited that OSS favors market expansion because of the openness of OSS organization of work, which generates knowledge from the community and thereby leading to a faster rate of innovation.

Furthermore, the Organisation for Economic Co-operation and Development [15] acknowledged that even though this positive effect of OSS and emerging technologies are self-evident in the overall economic growth of a nation, jobs and employment tend to shift from one section to another, typically from low-skilled jobs to high-skilled jobs. This also alludes to the earlier established "creative destruction" concept, where new technologies destroy old jobs (mostly low-skilled jobs) while creating new jobs requiring different and specialized skills. This is evident in an emerging technology like Autonomous Devices, where self-driving cars have the potential to create unemployment for taxi drivers and other logistics personnel, but at the same time, providing massive job opportunities for the programmers who develop the algorithms that run on such vehicles.

\section{THE REAL-WORLD IMPACT OF OPEN SOURCE ON EMERGING TECHNOLOGIES}

As has now been established, OSS presents certain characteristics that make it a powerful force for rapid innovation, while emerging technologies we further recognized have the potential for transforming existing markets and creating new ones which in turn yield economic growth. Right now, we specifically identify certain key emerging technologies, where the positive impacts of OSS have been felt.

\section{V.1 OPEN SOURCE IN ARTIFICIAL INTELLIGENCE AND MACHINE LEARNING}

Artificial Intelligence (AI) is a field of computer science involving the creation of computer systems that perform tasks such as learning, decision-making, reasoning, just like a human would do [16]. Machine Learning (ML) is a narrower field of AI which uses specialized algorithms to learn from large datasets, to learn, generalize, and predict. ML is one of the most heavily researched and technologically advanced branches of AI both in the industry and in academia. It also has seen lots of advancement due to the positive impact of OSS. For example, most of the current popular ML platforms supported by various IT giants are now open-source, such as Google Cloud Machine Learning Engine, TensorFlow by Google, Amazon's ML engine for AWS, Unity ML Agents, Apache PredictionIO, Microsoft Distributed Machine Learning Toolkit. These open-source ML engines are majorly the tools driving innovations in the industry with regards to developing and deploying ML models at scale.

For example, TensorFlow provides a comprehensive slew of tools, libraries, and community resources that provides the capability to develop and deploy state-of-the-art ML models, as easily as possible [17]. Also, Amazon provides a comprehensive collection of ML services that allows any developer or data scientist to build, train, and deploy ML services as easily as possible. This same open-source ML engine is what powers the plethora of Amazon's intelligent products such as Amazon Alexa, Amazon Prime Air, Amazon Go. Microsoft also provides its own open-source ML platform called Microsoft Distributed Machine Learning Toolkit, by using a distributed approach that allows multiple computers to solve complex ML problems in parallel to ensure scalability and flexibility [18]. Some other prominent opensource ML engines include PyTorch which is supported by Facebook, Apache MXNet which is supported by the Apache Software Foundation

All of these ML engines and toolkits provided by various IT giants, which are also currently being used at scale, could not have been made possible without the significant contributions made by open-source paradigms.

\section{V.2 OPEN SOURCE IN BLOCKCHAIN AND DISTRIBUTED LEDGER TECHNOLOGIES}

Blockchain is a decentralized electronic cryptographic ledger, where information called transactions, are distributed and synchronized across a network of computers, to enable decentralized generation, storage, and transfer of information [12]. The technology has garnered widespread research in both industry and academia, due to the interest in the key concepts, such as immutability, decentralization, on which the technology is founded upon. Some of the most commonly applied areas of blockchain include supply chain integration, financial services, digital identification and certification, and public records. OSS has also been able to greatly impact the development of Blockchain technology, as most of the prominent Blockchain platforms are open-source platforms.

For example, Ethereum, one of the most popular public Blockchain services currently available, featuring smart contract functionality, is an open-source project, which has largely received progress due to the contributions by the community. Also, Hyperledger Fabric, one of the most popular Blockchain systems mostly for enterprise-grade solutions, is a permissioned opensource project, hosted by the Linux Foundation. Some other common open-source Blockchain frameworks include the older and more common Bitcoin Blockchain and some other more highly efficient payment-based Blockchain networks like Ripple.

While Blockchain as a whole seems to be focused on payment and financial transactions, it should still be noted that it can be used as a cryptographically secure ledger to record anything of value, as it is currently being adopted in many other industry verticals. OSS is what is powering this rapid adoption and progress of Blockchain.

\section{V.3 OPEN SOURCE IN THE INTERNET OF THINGS} (IOT)

The Internet of Things (IoT) extends the global network concept of the Internet where only people communicate with themselves using computers, to a platform for devices to communicate electronically with themselves and the physical world around them [12]. This means that the plethora of data abounding around can be shared between devices and further used for other purposes. IoT is an umbrella word used to denote many other technologies that power it, some of which include, cloud computing, sensor networks, ubiquitous connectivity. IoT together with AI presents powerful capabilities in terms of bringing intelligence to the myriads of data used and shared with IoT devices and sensors. IoT has also been able to gain huge adoption because of the open-source nature of the platforms supporting it.

For example, Kaa IoT is an open-source, highly flexible cloud-based, multi-purpose, IoT middleware platform used to implement complete end-to-end IoT solutions, connected applications, and smart products. Thinger.io is an open-source IoT platform, making provisions for a scalable cloud infrastructure that connects devices together. Thinger.io also gives a high degree of 
control and flexibility as they provide RESTful APIs which users can use to integrate with the IoT services provided by the platform [19].

IoT concept is powerful as it is what is powering lots of 'smart initiatives', such as smart cities, smart manufacturing, smart agriculture, and it is the OSS concept that is truly powering this IoT movement.

\section{V.4 OPEN SOURCE IN MIXED REALITY, AUGMENTED REALITY, AND VIRTUAL REALITY}

Virtual Reality (VR) immerses users in a fully synthetic digital environment. Augmented reality (AR) overlays virtual objects in the real-world environment. Mixed reality (MR) combines both VR and AR by not just overlaying virtual objects, but also anchoring virtual objects to the real world. These three technologies in recent years have seen widespread interest most especially in education, military, fashion, sports, construction, media, telecommunications, films and entertainment, engineering, and healthcare applications. These technologies lack high adoption majorly as a result of the high cost of developing the software, and technical difficulties in implementing them, however, various IT giants like Facebook, Google, Microsoft, Magic Leap, HTC, Samsung are making their Software Development Kits (SDKs) open source to ease this barrier.

For example, ARToolKit is an open-source computer tracking library for the creation of strong augmented reality applications that overlay virtual imagery on the real world. Mixare (mix Augmented Reality Engine) is a free open-source augmented reality browser for Android and iOS platforms.

These technologies are immersive and crucial for certain industry verticals like healthcare and education but lack widespread adoption. Open-source software on the other hand is breaking the barriers to the adoption of these technologies by providing open-source SDKs and a community backing for these technologies.

\section{V.5 OPEN SOURCE IN AUTONOMOUS DEVICES}

Autonomous devices work with technologies such as AI and IoT to interact with their physical surroundings, which can further enable them to react based on the data gotten from their surrounding [12]. The most widely known autonomous device is the self-driving vehicle, which has the ability to navigate its surroundings partially or completely without human intervention. Although autonomous devices have received lots of interest, the adoption has also not been impressive, largely due to the high cost of developing the software, and technical difficulties in implementing them. However, open-source initiatives have facilitated more adoption of the technology.

For example, Apollo is an open-source, high-performance, flexible architecture that accelerates the development, testing, and deployment of Autonomous Vehicles. Autoware supports opensource projects enabling self-driving mobility, under the Autoware Foundation.

Open-source software projects are moving the bounds of what is possible in autonomous vehicles, and this is also encouraging a high adoption rate.

\section{THE REAL-WORLD IMPACT OF EMERGING TECHNOLOGIES ON ECONOMIC GROWTH}

Emerging technologies can bring about positive impacts on economic growth when adopted efficiently. Right now, we specifically identify certain emerging technologies that have contributed significantly to the economic growth of the country in which it was applied. We also identify the future impact of some of these emerging technologies on economic growth to demonstrate its wider positive impact.

As Çalışkan [20] identified, the real reason for the huge difference in the economic growth between the developed and developing countries is on the grounds of technological differences. Developed countries can effectively apply technology to reduce costs and while increasing productivity gains. Çalışkan [20] further argued that technological advancements make it possible to obtain more output with the use of the same quantity of input in any production process, hence bringing savings in the workforce and capital thereby further increasing the real Gross Domestic Product (GDP), which is a true indicator of economic growth.

Emerging technologies have the potential to disrupt existing markets and create new markets. This as already established is what underpins Schumpeter's creative destruction concept in which the emergence of new markets and the prevalence of new technologies becomes possible due to the "creative destruction" of the existing markets, which finally brings about innovation, structural changes, and economic growth [21].

\section{VI.1 ARTIFICIAL INTELLIGENCE, MACHINE LEARNING AND THE IMPACT ON ECONOMIC GROWTH}

Pilot implementation, investment, and research into AI are on the increase now more than ever. In a Deloitte study [22] it was discovered that the number of projects relying on ML doubled from 2017 to 2018, which was predicted to double again by 2020 . Furthermore, in a PwC report [23], it was revealed that AI had the real power to transform the productivity and GDP potential of the global economy. It was further predicted that by 2030, AI alone could contribute up to $\$ 15.7$ trillion to the global economy [23]. In another report by BusinessWire [24], AI was valued at $\$ 1.36$ billion in 2016 and was predicted to grow at a Compound Annual Growth Rate of 52\% from 2017 to 2025.

Albeit the potential for future growth, AI is currently already making positive grounds in many industry verticals in our today's world. For example, in healthcare, ML algorithms have been developed to diagnose some illnesses better than human experts [25]. Also, Deep Learning algorithms that can predict the onset of heart failure and hence prevent it, have been developed [26]. In supply chain and trade logistics, ML has been disrupting the flow of activities, reducing errors while yielding more productivity in the entire production and logistics process. In a study by Min [27], the intrinsic complexity of the supply chain was highlighted and AI was identified as a technological fit that can lead to end-to-end visibility across the supply chain while yielding better information management and better business decisionmaking. Also, the concept of supply chain 4.0 is currently making waves, where AI, along with other key emerging technologies like IoT, robotics, have been proposed to bring about tremendous automation in the supply chain operations and then finally yielding significant performance [28].

All of these are indicative of the transforming power of AI and ML, which, although still regarded as an emerging technology, is already bringing about positive results in the economies that can truly harness its powers. 


\section{VI.2 AUTONOMOUS VEHICLES AND THE IMPACT ON ECONOMIC GROWTH}

Autonomous Vehicles (AVs) like self-driving cars rides on the gains of $\mathrm{AI}$ and $\mathrm{ML}$, and it also has the potential to usher in a new age of economic growth. In a report by Mudge, et al. [29], it was predicted that the widespread adoption of AVs could yield significant economic benefits as about $\$ 800$ billion annual social and economic benefits could be realized by 2050, resulting from the reduction of vehicle crashes, productive time given back to commuters, and improved energy security by reducing dependence on oil which can also provide environmental benefits.

Although it should be noted that AVs will replace jobs with the automation of the rote activities involved in driving (meaning some people could lose their jobs), however, more jobs will be created requiring more specialized skills and knowledge. This also alludes to the previously established concept of creative destruction.

\section{VI.3 BLOCKCHAIN TECHNOLOGY AND THE IMPACT ON ECONOMIC GROWTH}

At the basic level, blockchain technology ensures the transfer of money, assets, and information in a secure manner via the Internet, in the absence of a third-party financial authority, by leveraging on cryptography, and finally storing these transactions as append-only tamper-resistant data structures. One key area Swan [30] was able to identify where blockchain technology can greatly impact was on Digital Asset Registries.

Digital Asset Registries leverage blockchain for the recording, transferring, and verification of asset ownership, for example, homes, cars, stocks, bonds, mortgages, and insurances, in addition to the preservation of the integrity and authenticity of sensitive documents or records such as passports, visas, driver's licenses, birth and death certificates, voter registration, contracts, wills, patents, and medical records. This is made possible by the cryptographic security present in blockchain technology. A good example to illustrate this is Sweden's blockchain-powered land registry, which has been estimated to save taxpayers over $\$ 106$ million yearly by the elimination of paperwork, fraud reduction, and the speeding up of transactions [31]. Swan [30] further identified blockchain technology as the technology truly capable of achieving global financial inclusion, ensuring that every unbanked person wherever they might be in the world, has access to financial services.

More so, about $58 \%$ of respondents in a survey of experts by the World Economic Forum in 2015 estimated that $10 \%$ of the world's GDP will be registered on a blockchain by the year 2025 [32].

All of these are indicative of the transformative power of blockchain technology in ensuring economic growth.

\section{VI.4 INTERNET OF THINGS AND THE IMPACT ON ECONOMIC GROWTH}

Internet of Things works together with $\mathrm{AI}$ and ML, cloud computing, sensor networks, ubiquitous connectivity to provide a global network of communicating devices. IoT is a technology that is currently impacting and will further greatly still impact consumers and industries due to the continuously increasing computing power and falling hardware prices.

McKinsey Global Institute [33] estimates that IoT will have a total potential economic impact of $\$ 3.9$ trillion to $\$ 11.1$ trillion per year in 2025 and they further suggest that IoT will contribute about $11 \%$ of total world GDP in 2025 . This alludes to the high potential of IoT in economic growth.

As has previously been identified, the main difference between the developed and developing nations is on the grounds of technological differences. The above-mentioned emerging technologies are just some of the technologies the developed nations are already relying upon for their incredible economic growth, even without leveraging the full potential of these technologies, as most of these technologies are still nascent.

\section{OPEN SOURCE SOFTWARE BUSINESS MODELS}

One nontrivial question to consider when dealing with open-source is, 'how do companies supporting open-source software make money so they do not go out of business?' This is because, as previously established, open-source software are freely used, modified, and distributed with respect to the copyright and license of its open-source status [5, 6], and hence, there must be a way for companies offering open-source software to make money. Right now, we identify some of the important business models behind some open-source projects, to show how companies behind such projects make profit out of it.

\section{VII.1 SUPPORT AND SERVICES}

Since open-source software itself is not sold, the support model involves selling crucial services such as technical support, deployment and integration services, consulting, training to businesses that want to deploy the software in a production scenario. With this, customers do not pay for the software itself, rather they pay for just support of the software if they so choose to. This gives another crucial advantage as customers can choose to switch to a similar software product if the current one does not satisfy their needs, as there is no payment for the software itself but just the services offered.

Another dimension taken by companies is to sell a packaged executable of their software to only customers who are willing to pay, while still leaving the source code open source. This way, the companies implicitly offer the service of compiling and packaging their source code as an executable binary. RedHat is one of the successful companies relying on this support and service model.

\section{VII.2 SOFTWARE AS A SERVICE (SAAS)}

In the SaaS model, the software is hosted on the cloud, which can be accessed from anywhere via a web browser. What makes the SaaS model work is the provision of subscriptions to more services by paying more money. What that means is that, although the base services of the software could be offered for free, the more advanced and most times useful services have to be paid for. SaaS models are flexible enough to give customers the ability to choose payment models that suits their needs. For example, a pay-as-you-go model is most common with the SaaS model subscriptions. WordPress is an example of a successful company relying on this model.

\section{VII.3 DUAL-LICENSING}

In this model, the software company maintains two separate license - an open-source license where the software is free and open, and a proprietary license requiring payments. The opensource license usually provides the basic functionalities that can facilitate testing, proof of concept development, and small-scale development, thereby enabling customers to understand the 
software to a reasonable extent. However, the more advanced features such as monitoring, administration, backup/restore as is in the case of database software programs, which are required for a production scenario, are reserved for a commercial license. MySQL database, a pioneer of this dual-licensing model, is an example of a software product relying on this model for profitmaking.

\section{VII.4 ADVERTISEMENT-BASED MODEL}

This model involves the commercialization of OSS by providing advertisement opportunities in such OSS. Many big companies such as Google, Mozilla profit from this model by providing avenues for other companies and businesses to advertise on their platforms for a fee. Another concept is the one done by AdBlock Plus, the popular open-source ad-blocking extension, where their profit comes from the company offering advertisers the ability to not have their ads blocked by their plugin by paying a fee for that.

\section{VII.5 PAID CERTIFICATION}

This model relies on the popularity of the software as if it is popular enough, certification opportunities could be offered for professionals who want to validate their skills and knowledge to advance their careers and stay relevant. Certifications provide a win-win situation as the companies offering them make more money, while the certification lends additional credibility to the professionals getting certified and most times even to the companies they work for. RedHat is also another open-source giant that relies on this model for profitmaking.

The above-mentioned business models are just some of the common models used by OSS companies to make profit from their software, as the list of business models is inexhaustive. Hence, even though OSS are freely used, modified, and distributed, tremendous profits can still be made out of it.

\section{CONCLUSION}

In this research, the concept of OSS was largely explored, where the major tenets of OSS such as collaboration, knowledge sharing, and volunteer software development, were investigated to see how OSS fosters innovation. Furthermore, emerging technologies, which have the potential to greatly disrupt existing markets and create new markets were also considered. It was then discovered that although OSS and emerging technologies are a powerful force for disruption and economic growth, their prevalence tends to cause a shift in the market, leading to new jobs requiring specialized skills, replacing the low-skilled old jobs. This as was highlighted had the effect of causing massive unemployment to some people, while at the same time, giving jobs to some other people - those possessing the right skillset. Finally, this research investigated the current strides made in emerging technologies due to the impact of OSS, which in turn increases economic growth. This led to the conclusion that OSS techniques should be encouraged, as it can lead to the rapid development of the economy of the country in which it is implemented.

\section{AUTHOR'S CONTRIBUTION}

Conceptualization: Eseosa Ehioghae and Sunday Idowu.

Methodology: Eseosa Ehioghae and Sunday Idowu.

Investigation: Eseosa Ehioghae and Sunday Idowu.

Discussion of results: Eseosa Ehioghae and Sunday Idowu.
Writing - Original Draft: Eseosa Ehioghae.

Writing - Review and Editing: Eseosa Ehioghae and Sunday Idowu.

Resources: Eseosa Ehioghae and Sunday Idowu.

Supervision: Sunday Idowu.

Approval of the final text: Sunday Idowu.

\section{REFERENCES}

[1] D. Rotolo, D. Hicks and B. R.Martin, "What is an emerging technology?," Research Policy, vol. 44, no. 10, pp. 1827-1843, 2015.

[2] M. Halaweh, "Emerging Technology: What is it?," Journal of Technology Management \& Innovation, vol. 8, no. 3, pp. 108-115, 2013.

[3] G. S. Day and P. J. H. Schoemaker, Eds., Wharton on Managing Emerging Technologies, 1st ed., Wiley, 2000.

[4] R. Ghafele and B. Gibert, "Open Growth: The Impact of Open Source Software on Employment in the USA," International Journal of Open Source Software and Processes (IJOSSP), vol. 5, no. 1, 2014

[5] C. Ebert, "Open Source Software in Industry," IEEE Software, vol. 25, no. 3 , pp. 52-53, 2008.

[6] FLOSS Manuals, "Practical Open Source Software Engineering," 2020 [Online]. Available: http://write.flossmanuals.net/practical-open-source-softwareengineering/introduction-to-foss/. [Accessed 25 October 2020].

[7] Red Hat, "What is open source?," 2020. [Online]. Available: https://opensource.com/resources/what-open-source. [Accessed 25 October 2020].

[8] C. Ebert, "Open Source Drives Innovation," IEEE Software, vol. 24, no. 3, pp 105-109, 2007.

[9] E. Raymond, "The cathedral and the bazaar," Knowledge, Technology \& Policy, vol. 12, pp. 23-49, 1999.

[10] A. Avila-Robinson and K. Miyazaki, "Conceptualization and Operationalization of Emerging Technologies: A Complementing Approach," in 2011 Proceedings of PICMET '11: Technology Management In The Energy-Smart World (PICMET), Portland, 2011.

[11] R. Adner and D. A. Levinthal, "The Emergence of Emerging Technologies," California Management Review, vol. 45, no. 1, pp. 50-66, 2002.

[12] R. D. Atkinson, "Emerging Technologies and Preparing for the Future Labor Market," Information Technology and Innovation Foundation (ITIF), 2018.

[13] A. Nayyar, "Top Emerging Open Source Technologies," 2018. [Online]. Available: https://www.opensourceforu.com/2018/03/ten-emerging-open-sourcetechnologies/. [Accessed 26 October 2020].

[14] G. Garzarelli, Y. R. Limam and B. Thomassen, "Open Source Software and Economic Growth: A Classical Division of Labor Perspective," Information Technology for Development, vol. 14, no. 2, pp. 116-135, 2008.

[15] Organisation for Economic Co-operation and Development, "Technology, Productivity and Job Creation: Best Policy Practices," Organisation for Economic Co-operation and Development.

[16] D. Castro and J. New, "The Promise of Artificial Intelligence," Center for Data Innovation, 2016

[17] TensorFlow, "TensorFlow," 2020. [Online]. Available: https://www.tensorflow.org/. [Accessed 30 October 2020].

[18] G. Thomas, "Microsoft open sources Distributed Machine Learning Toolkit for more efficient big data research," 2015. [Online]. Available: https://www.microsoft.com/en-us/research/blog/microsoft-open-sourcesdistributed-machine-learning-toolkit-for-more-efficient-big-data-research/. [Accessed 30 October 2020]

[19] thinger.io, "thinger.io," 2018. [Online]. Available: https://thinger.io/ [Accessed 30 October 2020].

[20] H. K. Çalışkan, "Technological Change and Economic Growth," Procedia Social and Behavioral Sciences, vol. 195, pp. 649-654, 2015. 
[21] M. Justman and M. Teubal, "A Structuralist Perspective on the Role of Technology in Economic Growth and Development," World Development, vol. 19, no. 9, pp. 1167-1183, 1991.

[22] Deloitte, "Machine learning: things are getting intense," Deloitte, London, 2017.

[23] PwC, "Sizing the prize What's the real value of AI for your business and how can you capitalise?," PwC, 2017.

[24] BusinessWire, "Global Artificial Intelligence Market 2017-2025 - Growth Trends, Key Players, Competitive Strategies and Forecasts Report - Research and
Markets,"
2017.
[Online].
Available:

https://www.businesswire.com/news/home/20171130005513/en/Global-ArtificialIntelligence-Market-2017-2025---Growth. [Accessed 1 November 2020].

[25] T. Kubota, "Stanford algorithm can diagnose pneumonia better than radiologists," 2017.2 [Online]. https://news.stanford.edu/2017/11/15/algorithm-outperforms-radiologistsdiagnosing-pneumonia/. [Accessed 1 November 2020].

[26] E. Choi, A. Schuetz, W. F. Stewart and J. Sun, "Medical Concept Representation Learning from Electronic Health Records and its Application on Heart Failure Prediction," Cornell University, Atlanta, 2016.

[27] H. Min, "Artificial intelligence in supply chain management: theory and applications," International Journal of Logistics: Research and Applications, vol. 13 , no. 1 , pp. 13-39, 2010

[28] K. Alicke, J. Rachor and A. Seyfert, "Supply Chain 4.0 - the next-generation digital supply chain," McKinsey \& Company, 2016.

[29] R. Mudge, D. Montgomery, E. Groshen, J. P. Macduffie, S. Helper and C. Carson, "America's Workforce and the Self-Driving Future: Realizing Productivity Gains and Spurring Economic Growth," Securing America's Future Energy (SAFE), 2018

[30] M. Swan, "Anticipating the Economic Benefits of Blockchain," Technology Innovation Management Review, vol. 7, no. 10, pp. 6-13, 2017.

[31] J. I. Wong, "Sweden's blockchain-powered land registry is inching towards reality," 2017. [Online]. Available: https://qz.com/947064/sweden-is-turning-ablockchain-powered-land-registry-into-a-reality/. [Accessed 1 November 2020].

[32] World Economic Forum, "Deep Shift - Technology Tipping Points and Societal Impact," World Economic Forum, 2015.

[33] McKinsey Global Institute, "The Internet of Things: Mapping the value beyond the hype," McKinsey \& Company, 2015. 